\title{
Factores de Riesgo para Enfermedad Cardiovascular en Trabajadores de una Institución Prestadora de Servicios de Salud, Colombia
}

\section{Cardiovascular disease risk factors in people working at a Colombian health institution}

\author{
Jesús E. Díaz-Realpe, Juliana Muñoz-Martínez y \\ Carlos H. Sierra-Torres
}

Facultad Ciencias de la Salud, Universidad del Cauca, Popayán, Colombia. jediaz@unicauca.edu.co, jmmunoz@unicauca.edu.co, hsierra@unicauca.edu.co

Recibido 2 Mayo 2006/Enviado para Modificación 19 Septiembre 2006/Aceptado 30 Noviembre 2006

RESUMEN

Objetivo Establecer la prevalencia de reconocidos factores de riesgo biológico y comportamental para enfermedad cardiovascular (ECV) en una población de trabajadores de una Institución Prestadora de Servicios de Salud (IPS) de la ciudad de Popayán, Colombia.

Métodos Se realizó un estudio observacional descriptivo, encuestando a 96 trabajadores para obtener información sobre características sociodemográficas, consumo de tabaco y alcohol, actividad física, hábitos alimentarios, y antecedentes personales y familiares para ECV. Además, a cada trabajador se le realizó medición estandarizada de glicemia, lípidos séricos, tensión arterial e índice de masa corporal. Resultados La prevalencia de factores de riesgo de tipo comportamental fue: tabaquismo $12,5 \%$, consumo de bebidas alcohólicas 58,3 \%, inactividad física en el tiempo libre $56,3 \%$, y dieta aterogénica $82,3 \%$. La prevalencia para factores de riesgo de tipo biológico fue: hipertensión arterial (HTA) 11,5 \%, sobrepeso 45,8 \%, diabetes $1 \%$, dislipidemia 61,5 \%, y antecedentes familiares de ECV 58,3\%. Adicionalmente, se encontró que el riesgo de HTA fue significativamente mayor en hombres, en individuos $>40$ años, en personas con un IMC $>25$, y en fumadores. En cuanto a la dislipidemia, el riesgo fue mayor en hombres y en individuos $>40$ años. Por su parte, el riesgo de sobrepeso y obesidad se asoció significativamente con el tabaquismo y el consumo de alcohol.

Conclusión En vista de la alta prevalencia de los factores de riesgo biológicos y ligados al comportamiento en la IPS de Popayán y aunque ésta es una institución de salud con una población asintomática y relativamente joven (25-55 años), se hace necesario tomar acciones encaminadas a modificar los estilos de vida para disminuir en forma importante el riesgo de ECV en la población afectada.

Palabras Clave: Prevalencia, factores de riesgo, enfermedades cardiovasculares, Colombia (fuente: DeCS, BIREME). 


\section{ABSTRACT}

Objective Establishing the prevalence of recognised biological and behavioural cardiovascular disease (CVD) risk factors in a population of workers at a health institution in Popayán, Colombia.

Methods An observational descriptive study was conducted, 96 employees being interviewed to obtain information regarding their socio-demographic characteristics, tobacco and alcohol consumption, physical activity, eating habits and personal and family history of CVD. Each worker's standardised glycaemia, serum lipids, arterial blood pressure and body mass index were measured.

Results There was $12,5 \%$ behavioural risk factor prevalence for smoking, 58,3 \% for alcohol consumption, 56,3\% physical inactivity during free time and $82,3 \%$ atherogenic diet; biological risk factor prevalence was $11,5 \%$ hypertension, 45,8 \% being overweight, $1 \%$ diabetes, $61,5 \%$ dyslipidaemia and $58,3 \%$ family history of CVD. It was also found that the risk of hypertension was significantly higher in males, in individuals $>40$ years, in people having $>25 \mathrm{BMI}$ and in smokers. Regarding dyslipidaemia, the risk was higher in males and in $>40$ year old individuals. The risk of being overweight and obese was significantly associated with smoking and alcohol consumption.

Conclusion Although this is an institution having an asymptomatic and relatively young population (25-55 years), action should be taken towards modifying lifestyles to reduce CVD risk in the affected population due to the high prevalence of biological and behavioural risk factors.

Key Words: Prevalence, risk factor, cardiovascular disease, Colombia (source: $\mathrm{MeSH}$, NLM).

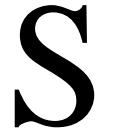

egún la Organización Mundial de la Salud, la enfermedad cardiovascular (ECV) es uno de los mayores problemas de salud pública en el mundo, siendo la primera causa de mortalidad al ocasionar 17 millones de muertes al año. La ECV es responsable de 32 millones de eventos coronarios y accidentes cerebro-vasculares, de los cuales entre el 40-70 \% son fatales en países desarrollados. Se estima que este problema es mucho mayor en países en vía de desarrollo y se considera que millones de personas padecen los factores de riesgo que no son comúnmente diagnosticados, tales como hipertensión arterial, tabaquismo, diabetes, hiperlipidemias y dieta inadecuada (1).

La ECV es incapacitante y costosa, ocasionando a nivel mundial 43 millones de años vividos con discapacidad y la pérdida de más de 147 millones de años de vida saludable (2). En Latinoamérica, esta entidad es responsable de 3 millones de años de discapacidad y de 9 millones de años de vida saludable (3). La ECV afecta a todos los estratos socioeconómicos, sin embargo, cerca del $85 \%$ sucede en países con bajos o moderados ingresos (1). En Colombia, la ECV es la segunda causa de muerte, siendo únicamente superada por la violencia (4). 
De manera alarmante, en la última década, la ECV ha venido incrementando como causa de morbilidad y mortalidad en países con bajos y medianos ingresos; en parte debido a los efectos de la globalización mediados por el mercadeo agresivo y las comunicaciones masivas que invitan a estas nuevas poblaciones a los estilos de vida de riesgo (tabaquismo, consumo de alcohol, dietas no saludables) (5).

El concepto de factor de riesgo de la ECV se aplica a aquellos signos biológicos y hábitos adquiridos que se han encontrado con mayor frecuencia entre los enfermos de cardiopatía en relación con la población general, permitiendo así la identificación de grupos poblacionales con mayor riesgo de presentar la enfermedad en los próximos años (6). En la actualidad los factores de riesgo para ECV han sido agrupados en modificables y no modificables. Los modificables son los que son susceptibles de cambiar bien sea mejorando el estilo de vida o con terapia farmacológica y los no modificables son aquellos imposibles de cambiar como la edad, el género y la herencia (7).

Estos factores de riesgo son responsables del $75 \%$ de la epidemia de ECV en el mundo; no obstante, la importancia de cada factor de riesgo es relativa y puede variar en las diferentes poblaciones (1). La identificación de estos factores y su distribución en la población es importante dado que existe evidencia de que al tomar acciones en contra de estos factores, el riesgo de ECV puede ser disminuido significativamente $(8,9)$.

Bajo este contexto, el objetivo del presente estudio fue establecer la prevalencia de reconocidos factores de riesgo para ECV en una población de trabajadores de una Institución Prestadora de Servicios de Salud (IPS) de la ciudad de Popayán, Colombia.

\section{MÉTODOS}

Se realizó un estudio observacional descriptivo de corte transversal, en los trabajadores de una IPS (Clínica de segundo nivel) que presta sus servicios médicos en la ciudad de Popayán, entre Octubre de 2004 a Mayo de 2005. Los criterios de inclusión fueron tener un tiempo de vinculación mayor o igual a 1 año en la Institución y reportar en la historia de salud ocupacional pruebas de perfil lipídico y glicemia del último semestre de 2004. Se invitaron a participar a todos los trabajadores de la institución $(\mathrm{n}=120)$, de los cuales $96(80 \%)$ aceptaron voluntariamente participar en el estudio y firmaron el consentimiento informado. El Comité de Ética para la Investigación Científica de la Universidad 
del Cauca, externo al grupo de investigación, revisó y aprobó la ejecución del presente estudio, acorde con la normatividad internacional y nacional existentes $(10,11)$.

Para la recolección de la información se diseñó un procedimiento con dos componentes. El primero fue un cuestionario que se aplicó a la historia de salud ocupacional para obtener los datos sobre pruebas de glicemia y perfil de lípidos, las cuales fueron procesadas por profesionales que laboran en el laboratorio de la misma institución, usando técnicas estandarizadas y validadas para tal fin. El segundo fue una encuesta estructurada, la cual se aplicó personalmente a cada individuo en el sitio de trabajo para indagar sobre variables sociodemográficas, antecedentes familiares y personales de enfermedad cardiovascular, hábito de fumar, consumo de bebidas alcohólicas, actividad física, y dieta en el último año.

Se consideró como "fumador" al consumidor de al menos 1 cigarrillo al día, como "exfumador" a la persona que había dejado de fumar en un tiempo mayor o igual a 6 meses anteriores a la encuesta, y como "no fumador" a las personas que declararon el no consumo de tabaco en el momento de la encuesta. Para el consumo de alcohol se considero bajo, si la ingesta era de una a dos veces por mes de 6 y 10 tragos; moderado, si la ingesta era de una vez a la semana de 1 a 10 tragos; o alto, si la ingesta era de dos o mas veces a la semana y mas de 10 tragos (12). Se consideraron físicamente "inactivas" todas aquellas personas que realizaban menos de 150 minutos de actividad física semanal; aquellos que igualaron o superaron ese nivel se consideraron físicamente "activos" (13).

Durante la entrevista, se estableció la tensión arterial utilizando tensiómetros de mercurio calibrados en el brazo derecho con la persona sentada y en reposo; considerándose hipertenso a todos aquellos sujetos con tensión arterial sistólica mayor o igual a 140, tensión arterial diastólica tensión arterial diastólica mayor o igual a 90 mmHg, o con antecedente de diagnóstico médico para hipertensión arterial. El índice de masa corporal (IMC) se calculó con base en la talla y el peso medidos con tallímetros y báscula calibrados, según la formula IMC = Peso (Kg)/Talla $\left(\mathrm{m}^{2}\right)$, y se consideró sobrepeso a todas las personas con IMC mayor que 25, incluyendo así preobesos y obesos. Se consideró a una persona diabética si la glicemia tomada en ayunas era mayor que $124 \mathrm{mg} / \mathrm{dl}$. En cuanto al perfil lipídico, se consideraron como valores normales aquellos menor que $200 \mathrm{mg} / \mathrm{dl}$ de colesterol total, menor que $150 \mathrm{mg} / \mathrm{dl}$ de triglicéridos, y valores mayor que $35 \mathrm{mg} / \mathrm{dl}$ en hombres y mayor que $45 \mathrm{mg} / \mathrm{dl}$ en mujeres para 
colesterol HDL. Se consideró dislipidemia en aquellos individuos que presentaron una o más alteraciones en el perfil lipídico $(14,15)$.

Para establecer los hábitos alimenticios se realizó una anamnesis alimentaría en la que se tenía en cuenta el consumo diario, semanal y ocasional de los alimentos en el ultimo año. El análisis nutricional se hizo teniendo como referencia la tabla de composición de alimentos y los resultados se asimilaron a las recomendaciones de kilocalorías y nutrientes para la población colombiana (16). La dieta se consideró aterogénica si existía un consumo alto de grasas saturadas, superando en dos veces el consumo de las grasa polinsaturadas, consumo alta de azúcares simples y refinadas, y un consumo bajo de fibra.

Para el procesamiento y análisis estadístico de los datos, se construyó una base de datos en SPSS versión 13.0 para Windows (SPSS Inc., Chicago, IL, EUA). Las variables continuas se expresaron con la media \pm desviación estándar y las variables discretas se expresaron en frecuencias y proporciones. Posteriormente, se realizó un análisis bivariado para determinar la asociación entre las variables sociodemográficas y comportamentales con el riesgo relativo (OR) de hipertensión arterial (HTA), dislipidemia, y sobrepeso y obesidad. Además, se calcularon los intervalos de confianza del 95 \% (IC $95 \%$ ) para cada OR y se ajustaron por edad y sexo.

\section{RESULTADOS}

De los 120 trabajadores invitados a participar en el estudio, un total de 96 (80 \%) fueron reclutados voluntariamente. Los 96 participantes tenían el 100\% de la información solicitada en el cuestionario.

En la Tabla 1 se indican las características sociodemográficas de la población a estudio. El promedio de edad ( \pm desviación estándar) fue de 41,17 $\pm 6,57$ años, con un rango entre los 25 y 55 años, y el mayor grupo de población estuvo entre los 41 y 50 años (52 \%). En cuanto al género, hubo predominio del género femenino, con una razón mujer/hombre de 2,3:1. La mayoría de la población tenía pareja, predominando el matrimonio (37/56) y la unión libre (19/56), y el mayor grupo de población se concentró en el estrato socioeconómico III (59,5 \%). Como era de esperarse para una institución de salud, la mayoría de la población presentó un grado de escolaridad mayor o igual que secundaria completa (90,6 \%). El 94,8 \% de la población no tenía antecedentes personales de ECV, 3,1 \% habían presentado accidente cerebro 
vascular y 2,1 \% otro tipo de ECV. De otro lado, el 58,3 \% de la población reportó antecedentes familiares de ECV.

Tabla 1. Características sociodemográficas

\begin{tabular}{lrr} 
& Características & \\
& Frecuencia & $\%$ \\
\hline Edad (años) & 6 & 6,3 \\
$20-30$ & 34 & 35,4 \\
$31-40$ & 50 & 52,0 \\
$41-50$ & 6 & 6,3 \\
$51-60$ & & \\
Sexo & 29 & 30,2 \\
Masculino & 67 & 69,8 \\
Femenino & & \\
Estado conyugal & 56 & 58,3 \\
Con pareja & 40 & 41,7 \\
Sin pareja & & \\
Estrato socioeconómico & 1 & 1,0 \\
I & 3 & 3,1 \\
II & 57 & 59,5 \\
III & 25 & 26,0 \\
IV & 10 & 10,4 \\
V & & \\
Escolaridad & 10 & 10,4 \\
Secundaria incompleta & 30 & 31,3 \\
Secundaria completa & 28 & 29,2 \\
Estudios tecnológicos & 23 & 24,0 \\
Estudios universitarios & 5 & 5,1 \\
Postgrado & 56 & 5,2 \\
Antecedentes de enfermedad cardiovascular & 58,3 \\
Personales & 56 \\
Familiares & & \\
\hline
\end{tabular}

En la Tabla 2 se muestra la prevalencia de los factores de riesgo. De las 96 personas encuestadas, 12 (12,5\%) reportaron ser fumadores, de los cuales 6 eran hombres y 6 mujeres; pero la proporción de fumadores fue mayor en los hombres en comparación a las mujeres, correspondiendo a 20,7 \% (6/29) y 9,0 $\%$ (6/67), respectivamente. Se encontró una proporción importante de consumo de alcohol (58,3\%), es de resaltar que el mayor porcentaje eran consumidores de bajas cantidades (39,6 \%). En cuanto a actividad física la proporción de activos e inactivos fue similar. Un alto porcentaje de individuos consumían una dieta aterogénica (82,3\%), dada principalmente por un consumo elevado de grasas saturadas, azúcares refinados y un bajo consumo de grasas poliinsaturadas y fibra. La prevalencia de HTA fue de $11,5 \%$, correspondiente a un $24 \%$ (7/29) en hombres y un 6 \% (4/67) en mujeres. Con relación al IMC, el 43,8 \% de la población presentó sobrepeso, correspondiendo a 17/29 hombres y 25/67 mujeres. La prevalencia de diabetes fue del 1 \% y correspondió a una 
mujer. El 51 \% de la población presentó hipercolesterolemia, de los cuales 19/ 29 fueron hombres y 30/67 mujeres. Un 40,6 \% de la población presentó hipertrigliceridemia, siendo 17/29 hombres y 22/67 mujeres. El HDL no protector estuvo presente en 31,3 \% de la población, correspondiendo a 4/29 hombres y 26/67 mujeres. En total, se encontró un 75 \% de la población con dislipidemia, correspondiendo a 25/29 hombres y 47/67 mujeres.

Tabla 2. Prevalencia de factores de riesgo

\begin{tabular}{lrr}
\hline Factor & Frecuencia & $\%$ \\
\hline Tabaquismo & 12 & 12,5 \\
Fumadores & 84 & 87,5 \\
No fumadores & & \\
Consumo de alcohol & 40 & 41,7 \\
No consumen & 56 & 58,3 \\
Consumidores & 38 & 39,6 \\
Bajo & 16 & 16,7 \\
Moderado & 2 & 2,0 \\
Alto & & \\
Actividad física & 42 & 43,8 \\
Activo & 54 & 56,3 \\
Inactivo & & \\
Dieta & 79 & 82,3 \\
Aterogénica & 17 & 17,7 \\
No aterogénica & & \\
Tensión arterial & 85 & 88,5 \\
Normotenso & 11 & 11,5 \\
Hipertenso & & \\
IMC & 54 & 56,3 \\
Normal & 42 & 43,8 \\
Sobrepeso y obesidad & & \\
Glicemia & & 99,0 \\
No diabético & 1 & 1,0 \\
Diabético & & \\
Perfil lipídico & 49 & 51,0 \\
Hipercolesterolemia & 39 & 40,6 \\
Hipertrigliceridemia & 30 & 31,3 \\
HDL no protector & 72 & 75,0 \\
Dislipidemia & & \\
\hline
\end{tabular}

En la Tabla 3 se presentan los resultados de la asociación entre algunas variables sociodemográficas y comportamentales con el riesgo (OR) de HTA, dislipidemia, y sobrepeso y obesidad. Los valores de probabilidad $(p)$ indican aquellos OR que fueron significativos. Como se observa en la Tabla, el riesgo de HTA fue mayor en los hombres en comparación a las mujeres, en los individuos mayores de 40 años, en las personas con baja escolaridad (menor o igual que secundaria), en los fumadores, y en aquellos con un IMC superior a 25. En cuanto a la dislipidemia, se encontró un mayor riesgo en individuos mayores de 40 años y con baja escolaridad. Por su parte, el riesgo de sobrepeso 
y obesidad se asoció con la baja escolaridad y el consumo de cigarrillo.

\section{DISCUSIÓN}

En el presente estudio se muestra una alta prevalencia de factores de riesgo comportamentales y biológicos para ECV. Entre los factores de riesgo no modificables de mayor prevalencia estuvieron el sexo masculino y la edad (>40 años); entre los factores de riesgo modificables de tipo comportamental estuvieron el consumo de alcohol, la inactividad física y la dieta aterogénica; y entre los modificables de tipo biológico estuvieron la dislipidemia y el sobrepeso y la obesidad. El estudio costarricense de Hernández muestra que un 77,6 \% de la población tienen dos o más factores de riesgo para ECV (17), datos muy semejantes a los de este trabajo y a los descritos por otros investigadores colombianos $(6,18)$. De igual manera, los resultados del presente estudio son similares a los reportados en la literatura científica internacional por el proyecto MONICA (19).

Aunque la prevalencia de fumadores en el presente estudio fue bastante baja (12,5\%), el tabaquismo estuvo asociado con el riesgo de HTA (OR=4,0, IC95\%=0,85-18,52). La prevalencia de tabaquismo en este estudio fue menor a la reportada en el estudio del Carmen de 26,9\% (20) y en el estudio ENFREC II de 22,7 \% (21). En el estudio MONICA, la proporción de fumadores varía entre 34 y $64 \%$ en hombres y entre 3 y $52 \%$ en mujeres (19). En cuanto al consumo de alcohol, 18,7 \% de la población a estudio reporto un consumo entre moderado y elevado de bebidas alcohólicas, siendo inferior al informado en el estudio del Carmen (37,4 \%) en Colombia (20) y superior al reportado por Bustos y col. en Chile (13,2 \%) (22).

La prevalencia de inactivos en el tiempo libre fue de 56,3 \%, cifra menor que la informada en el estudio del Carmen de 68,8 \% (20) y mayor a la encontrada en el estudio del hospital San Ignacio de 43,7 \% (23).

La prevalencia de HTA (11,5\%) en el presente estudio es inferior a la informada por otros estudios en Colombia como ENFREC II (14 \%) (21) y el estudio del Carmen (16,7 \%) (20). Sin embargo, esta podría ser explicado por que en la población estudiada la edad osciló entre los 25 y 55 años y se conoce que la mayor prevalecía de HTA se encuentra en personas por encima de los 55 años; según la OMS la prevalencia de HTA varía entre el 15 al 37 \% en la población adulta (1). 


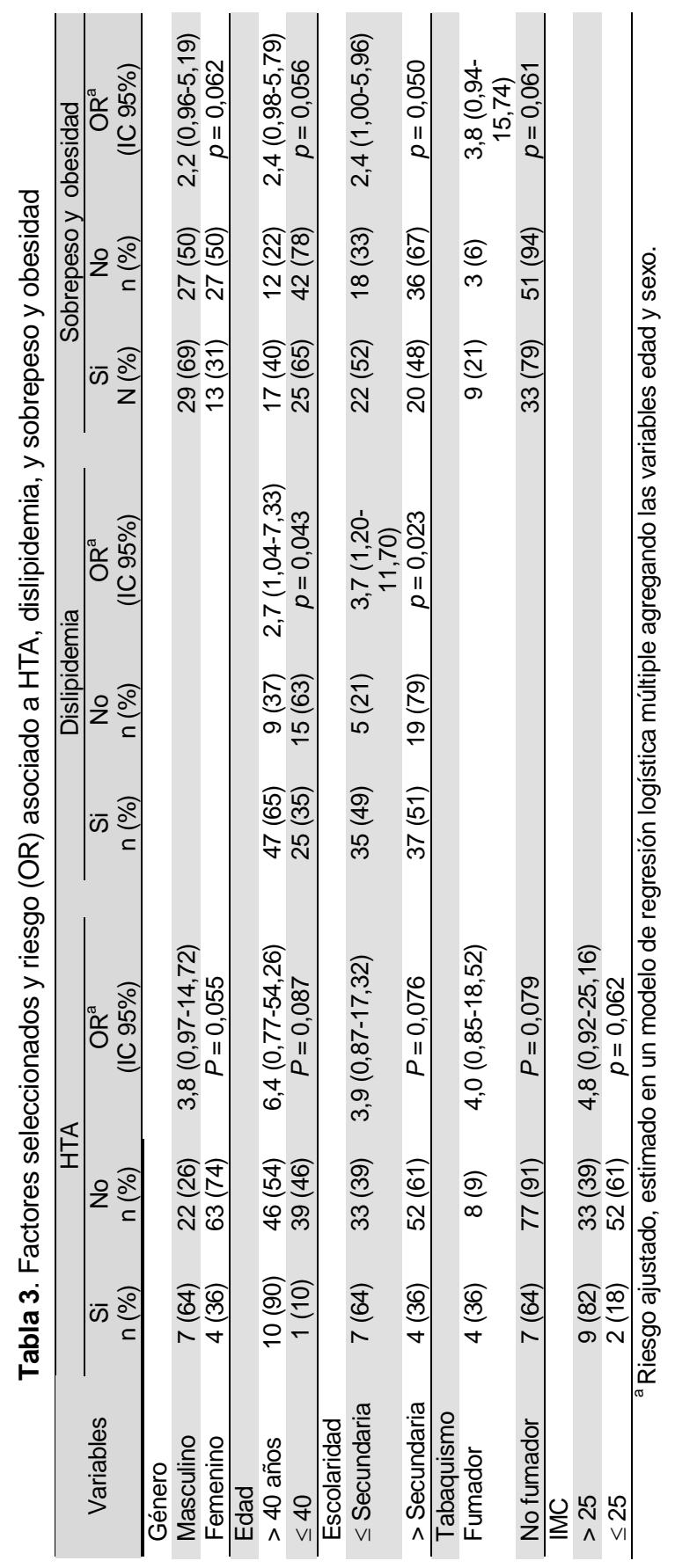


La prevalencia de dislipidemia (75 \%) y de hipercolesterolemia (51\%) encontradas en el presente estudio son más elevadas que los reportadas en el Hospital San Ignacio (23) y en la población del Carmen (20), estudios realizados en Bogotá. La prevalencia tan elevada de dislipidemia pudiera ser explicada por el consumo de una dieta aterogénica (82,3 \%) en la población a estudio.

La prevalencia de sobrepeso u obesidad reportada en este estudio (43,8 \%) es similar a estudios realizados en Colombia los cuales reportaron $42 \%$ (23) y 43,3 \% (20), y en Chile que reporta 47,7 \% (22). Actualmente, la prevalencia de obesidad va en ascenso en la mayoría de países y es considerada como una epidemia global, con graves consecuencias para la salud publica del mundo (24). El estudio Framingham ha documentado que la obesidad es un factor de riesgo independiente para ECV (25).

En el presente estudio, un mayor riesgo para desarrollar HTA estuvo asociado con las variables sexo masculino $(\mathrm{OR}=3,8)$, edad $>40$ años (OR $6,4)$, escolaridad $\leq$ secundaria $(\mathrm{OR}=3,9)$, tabaquismo $(\mathrm{OR}=4,0)$ y sobrepeso $\mathrm{u}$ obesidad $(\mathrm{OR}=4,8)$. Además, se presento una asociación significativa entre edad $>40$ años (OR 2,7) y escolaridad $\leq$ secundaria $(\mathrm{OR}=3,7)$ y un mayor riesgo de dislipidemia. Para obesidad y sobrepeso se encontró una asociación significativa con la escolaridad $\leq$ secundaria $(\mathrm{OR}=2,4)$ y el tabaquismo $(\mathrm{OR}=3,8)$. Es de resaltar que la HTA, la dislipidemia y el sobrepeso y la obesidad están entre los principales factores de riesgo para ECV (1). A pesar de que el tamaño de muestra en este estudio es relativamente pequeño, estas asociaciones son consistentes con otros estudios de gran magnitud como el proyecto MONICA (19).

Es muy posible, que las diferencias de las poblaciones analizadas en los diferentes estudios limite sustancialmente la comparación de los hallazgos. Sin embargo, este estudio podría ser el punto de partida para emprender trabajos a nivel poblacional que no tengan las limitaciones en cuanto a tamaño de muestra de los estudios institucionales. Además, los resultados aquí encontrados deben servir para la formulación de nuevas estrategias de intervención tanto a nivel primario como secundario para reducir la prevalencia de factores de riesgo para ECV en la población afectada

Agradecimientos. Los autores expresan su agradecimiento a las directivas y los trabajadores de la Clínica Antonio Nariño del Instituto de Seguros Sociales en la ciudad de Popayán, por su participación y colaboración en la ejecución del presente estudio. 


\section{REFERENCIAS}

1. World Health Organization. The World Health report 2002: reducing risks, promoting healthy life. Geneva, Switzerland: WHO; 2002. p. 1-230.

2. Anónimo. Nuevos factores de riesgo en cardiopatía isquémica. Tribuna Médica 2004; 104:2-3.

3. Rodríguez J, Gallardo HM. La carga de la enfermedad en Colombia 1985-1995. Bogotá: Ministerio de Salud, República de Colombia; 1998. p. 60-70.

4. Pan American Health Organization. Colombia Health Profile. In: Health in the Americas Vol. II, ed. PAHO. Washington, D.C.: PAHO; 1998. p. 181-193.

5. Advisory Board. The Milan declaration: positioning technology to serve global heart health. 5th International Heart Health Conference. Milan, Italy: WHO; 2004. p. 1-32.

6. Manzur F, Arrieta CO. Estudio sociológico y del conocimiento de los factores de riesgo de las enfermedades cardiovasculares en la Costa Caribe Colombiana (Estudio Caribe). Rev Colomb Cardiol 2005; 12:122-8.

7. Pearson TA, Blair SN, Daniels SR, Eckel RH, Fair JM, Fortmann SP et al. AHA guidelines for primary prevention of cardiovascular disease and stroke. Circulation 2002; 106:388-91.

8. Bakhru A, Erlinger TP. Smoking cessation and cardiovascular disease risk factors: results from the Third National Health and Nutrition Examination Survey. PLoS Med 2005; 2:e160.

9. Magnus P, Beaglehole R. The real contribution of the major risk factors to the coronary epidemics: time to end the "only-50\%" myth. Arch Intern Med 2001; 161:265760 .

10. World Medical Association. The Helsinki declaration. Orv Hetil 1965; 106:1715-6. 11. Ministerio de Salud. Resolución No. 8430. Santafé de Bogotá, D.C.: Ministerio de Salud, República de Colombia; 1993. p. 1-13.

12. Davidson R, Raistrick D. The validity of the Short Alcohol Dependence Data (SADD) Questionnaire: a short self-report questionnaire for the assessment of alcohol dependence. Br J Addict 1986; 81:217-22.

13. U.S. Department of Health and Human Services. Physical activity and health: A report of the surgeon general. Atlanta, USA: U.S. Department of Health and Human Services; 1996. p. 85-151.

14. Sociedad Colombiana de Cardiología. I consenso nacional para el diagnostico y manejo de la hipertensión arterial sistémica. Clínicas Colombianas de Cardiología 1998; 1:115-75.

15. Sociedad Colombiana de Cardiología. I consenso nacional sobre detección, evaluación y tratamiento de las dislipoproteinemias en adultos. Clínicas Colombianas de Cardiología 1998; 1:177-268. 
16. Instituto Colombiano de Bienestar Familiar. Tabla de composición de alimentos colombianos. Bogotá: Ministerio de Salud Pública, República de Colombia; 1978. p. 1-95.

17. Hernández WI. Factores de riesgo de enfermedad cardiovascular en una población obrera industrial de la provincia de Cartago. Rev Costarric Salud Pública 2000; 9:55-64.

18. Barrera E, Ceron N, Ariza MC. Conocimientos y factores de riesgo cardiovascular y su relación con la presencia de hipertensión arterial. Colomb Med 2000; 31:202.

19. Gregor RD, Bata IR, Eastwood BJ, Wolf HK. Ten-year trends of heart disease risk factors in the Halifax County MONICA population. MONItoring of trends and determinants in CArdiovascular disease. Can J Cardiol 1998; 14:1017-24.

20. Gómez LF, Samper B, Cabrera G, Espinosa G, Mateus JC, Gómez LC. Factores de riesgo cardiovascular en la localidad de Santa Fe de la ciudad de Bogotá. Resultados obtenidos en el área demostrativa Carmen. Boletín Epidemiológico Distrital 2004; 9:4-13.

21. Ministerio de Salud. III Estudio nacional de Salud Bucal ENSAB III-II. Estudio nacional de factores de riesgo de enfermedades crónicas - ENFREC II. Tomo II Tabaquismo. Bogotá, D.C.: Ministerio de Salud, República de Colombia; 1999. p. 48-76.

22. Bustos P, Amigo H, Arteaga A, Acosta A, Rona RJ. Factores de riesgo de enfermedad cardiovascular en adultos jóvenes. Rev Med Chile 2003; 131:973-8.

23. Díaz-Perilla M, Cordoba DP. Identificacion de factores de riesgo de enfermedad cardiovascular presentes en los pacientes que ingresan al Hospital San Ignacio. Lect Nutr 2003; 10:51-8.

24. James PT. Obesity: the worldwide epidemic. Clin Dermatol 2004; 22:276-80.

25. Hubert HB, Feinleib M, McNamara PM, Castelli WP. Obesity as an independent risk factor for cardiovascular disease: a 26-year follow-up of participants in the Framingham Heart Study. Circulation 1983; 67:968-77. 